
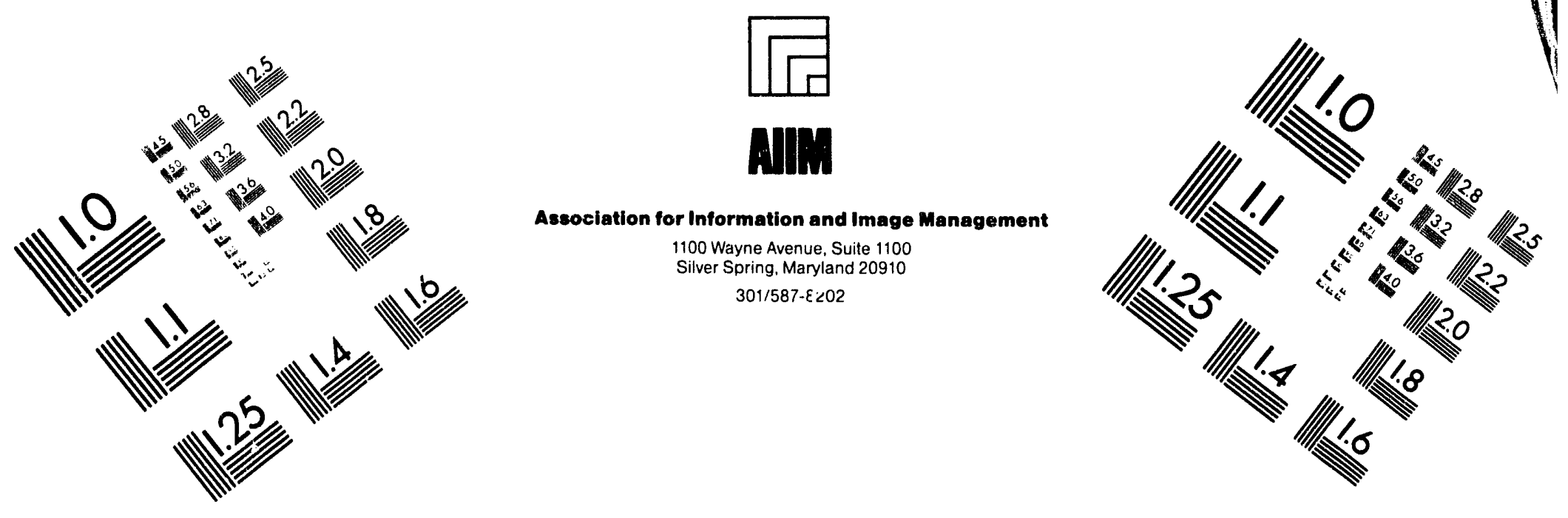

\title{
Centimeter
}

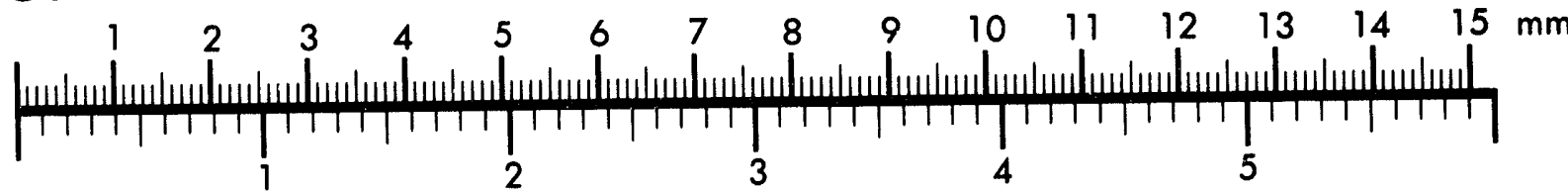

Inches
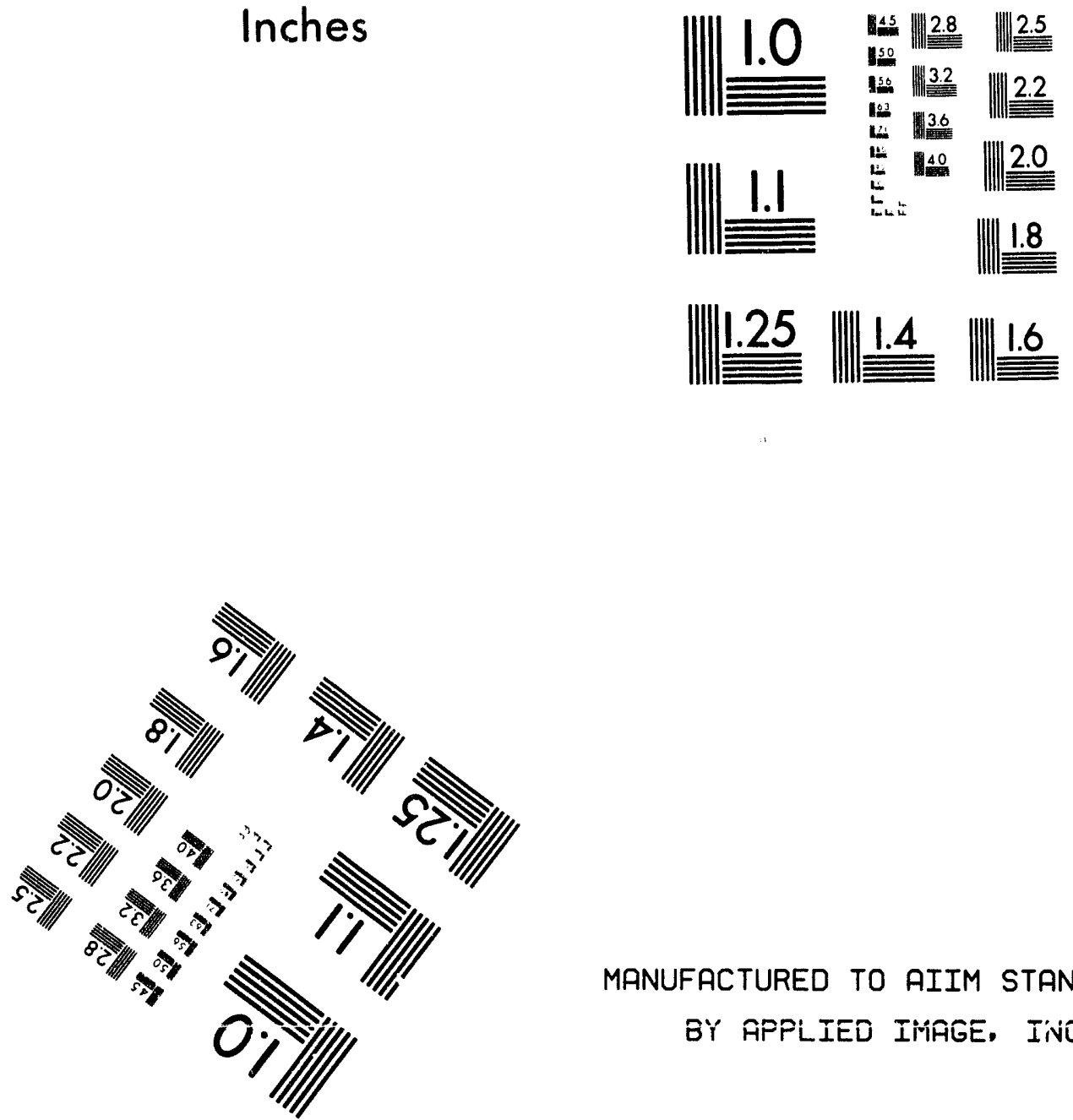

MANUFACTURED TO AIIM STANDARDS

BY GPPLIED IFIAGGE, INVC.

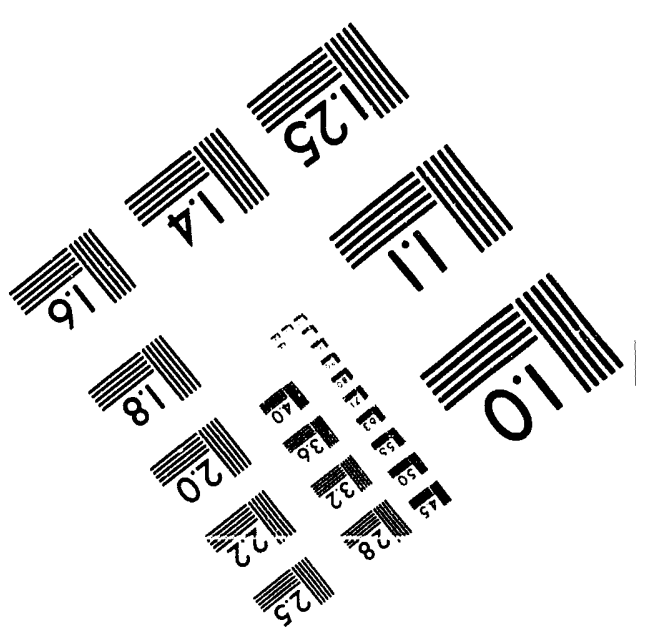



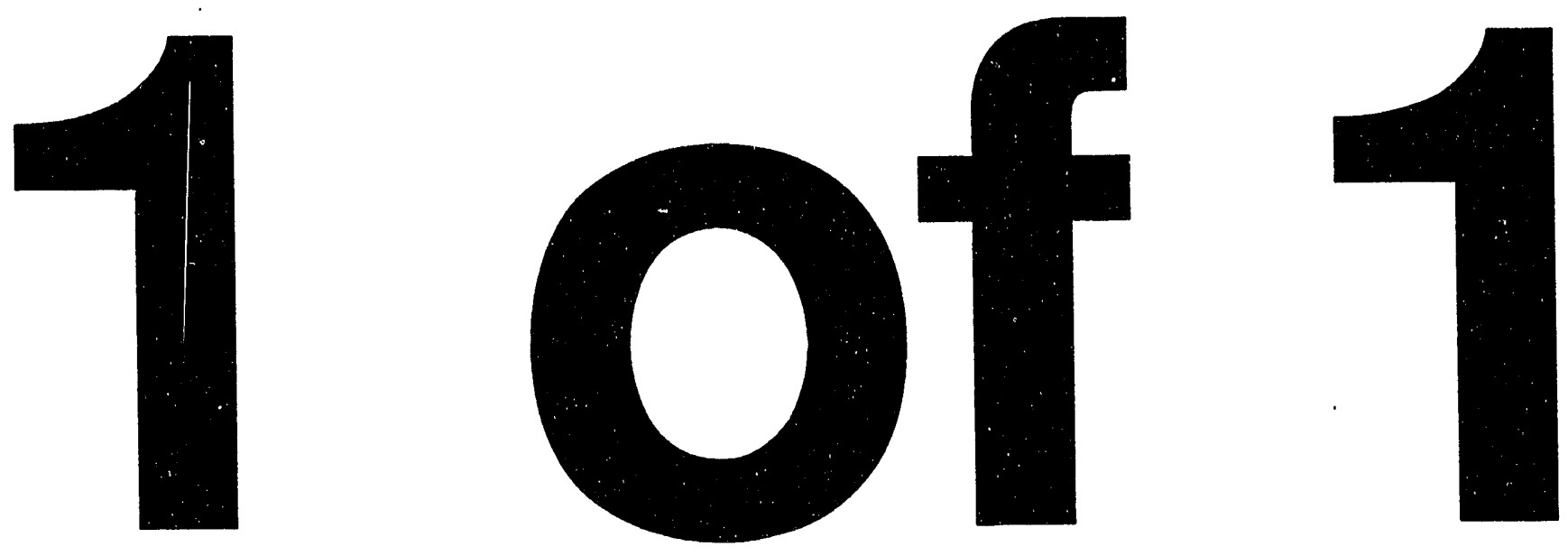


\title{
$\cos (f-9302191-15$
}

\section{ENVELOPE MODEL OF BEAM TRANSPORT IN ILSE}

UCRL-JC-112244

PREPRINT

\author{
W. M. Sharp, J. J. Barnard, D. P. Grote, S. M. Lund, and S. S. Yu ${ }^{\dagger}$
}

Lawrence Livermore National Laboratory, University of California

P.O. Box 5508, L-440, Livermore California 94550, USA

${ }^{\dagger}$ Lawrence Berkeley Laboratory

Berkeley California 94720, USA

This paper was prepared for submittal to the

Proceedings of the Computational Accelerator Physics Conference

Pleasanton, California

February 22-26, 1993

March 23, 1993

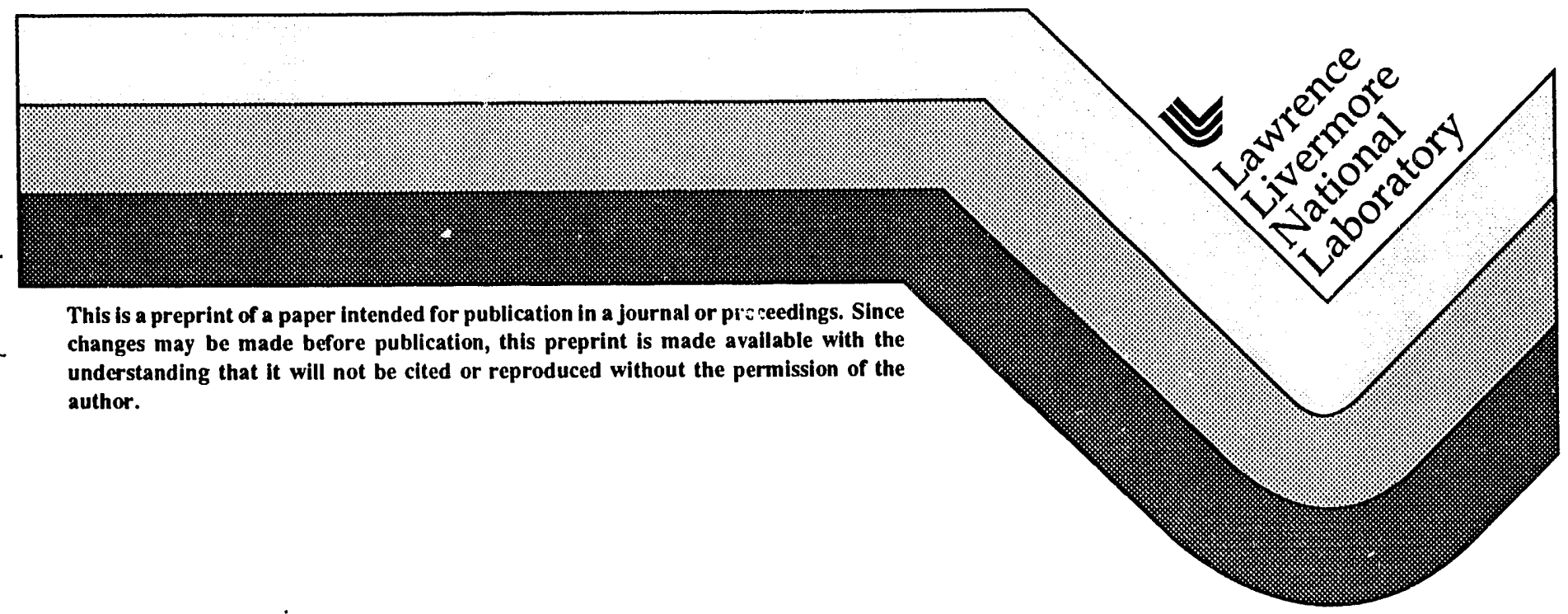




\section{DISCLAIMER}

This document was prepared as an account of work sponsored by an agency of the United States Govemment. Neither the United States Government nor the University of Califomia nor any of their employees, makes any warranty, express or implied, or assumes any legal liability or responsibility for the accuracy, completeness, or usefulness of any information, apparatus, product, or process disclosed, or represents that its use would not infringe privately owned rights. Reference herein to any specific commercial products, process, or service by trade name, trademark, manufacturer, or otherwise, does not necessarily constitute or imply its endorsement, recommendation, or favoring by the United States Government or the University of Califomia. The views and opinions of authors expressed herein do not necessarily state or reflect those of the United States Govemment or the University of Califomia, and shall not be used for advertising or product endorsement purposes. 


\title{
ENVELOPE MODEL OF BEAM TRANSPORT IN ILSE*
}

\author{
W. M. Sharp, J. J. Barnard, D. P. Grote, S. M. Lund, and S. S. Yu ${ }^{\dagger}$ \\ Lawrence Livermore National Laboratory, Livermore, CA 94550
}

\begin{abstract}
CIRCE is a fast-running beam dynamics code developed to facilitate the design and analysis of heavy-ion accelerators. The code combines an envelope description of the beam transverse dynamics with a fluidlike treatment of longitudinal dyuamics, and terms are included to account for the effects of space charge, emittance, and image forces. CIRCE is currently being adapted to model the Induction Linac Systems Experiments (ILSE) facility, a proposed heavy-ion accelerator designed to test aspects of an inertial-fusion driver. The numerical model in the code is discussed, and changes needed for modeling ILSE are outlined. Preliminary work is presented on beam matching along the ILSE lattice and on transport around the ILSE achromatic bend.
\end{abstract}

\section{INTRODUCTION}

The Induction Linac System Experiments (ILSE) is an ion accelerator and series of experiments ${ }^{1}$ planned at Lawrence Berkeley Laboratory to study critical physics issues in heavy-ion fusion (HIF) driver. The facility will accelerate four beams simultaneously from an injection energy of $2 \mathrm{MeV}$ to $5 \mathrm{Mev}$, using electrostatic quadrupoles for transverse focusing. After the four beams are merged in a "combiner," the single beam will be accelerated to $10 \mathrm{MeV}$. During acceleration, the beam will be compressed from an initial duration of $1 \mathrm{~ms}$ to $0.4 \mathrm{~ms}$ by imposing a velocity "tilt" along the beam length, with ions at the beam tail being accelerated more than those near the head. Among the planned experiments are a $180^{\circ}$ achromatic bend and a final section where the the beam is compressed as it drifts. The planned current and emittance of the ILSE beams are chosen to give the same line-charge density and beam diameter as in a HIF driver, making ILSE a realistic test of the dynamics of space-charge dominated beams.

The ILSE facility requires detailed design of the acceleration schedule. The beam must remain matched to the transverse focusing, and the strong longitudinal space-charge forces near the beam ends must be balanced by external fields as the beam is simultaneously accelerated and compressed. Due to the large parameter space, much of the initial design work will likely be done using the fast-running multiple-slice envelope code CIRCE ${ }^{2}$. The CIRCE model uses an envelope description of the beam transverse dynamics and treats the beam as a Lagrangian fluid in the longitudinal direction. Appropriate terms are included to account for the effects of image forces, beam emittance, and space-charge in the limit of paraxial motion, and the beam is focused and accelerated by a user-specified lattice of electrostatic and magnetic quadrupoles, bending magnets, and acceleration modules. Although the code was described in Ref. 1, the equations are extensively reviewed in Section II because numerous refinements and generalizations have been made since that publication. Further code modifications required for modeling ILSE are then presented in Section II along with preliminary simulations of the ILSE acceleration section and achromatic bend.

\section{MODEL}

The set of envelope equations used here to model the beam transverse dynamics is a generalization of that formulated first by Kapchinskij and Vladimirskij and later adapted by Lee, Close, and Smith. ${ }^{2}$ The beam transverse distribution function is assumed to be uniform and elliptical in each phase-space plane, and the coordinate-space ellipse may be taken to be unskewed provided that the quadrupoles have the same orientation everywhere and there is no axial magnetic field. Except for image forces, the treatment is first order in the ratio of the beam radii and centroid displacements to the beam-pipe radius. This approximation makes it appropriate to represent bend magnets and quadrupoles by idealized expressions and to neglect

* This work was performed under the auspices of the US Department of Energy by Lawrence Livermore National Laboratory under W-7405-ENG-48.

$\dagger$ Present address: Lawrence Berkeley Laboratory, Berkeley, CA 94720 


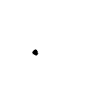


higher-order multipole fields. In the present version of the code, only single-function magnets are used, and a simple analytic model may optionally be used to represent the axial fringe fields. Although the ion mass is multiplied where it appears by the Lorentz factor $\gamma$, derivatives of $\gamma$ are small enough to be dropped from the ion-motion equations. Also, we assume a circular beam pipe of infinite conductivity and radius $R$.

\section{Transverse Dynamics}

With these assumptions, coupled envelope equations for the coordinate-space radii are found by differentiating the expressions defining the root mean-squared beam radii. We use a local Cartesian coordinate system centered on the beam-pipe axis, in which the coordinate $s$ is distance around the axis of the lattice, and $x$ and $y$ are respectively the spatial coordinates in and perpendicular to the nominal plane of the lattice. For a beam pipe with a local bend radius $\rho$ and a curvature vector that is rotated in the transverse plane by an angle $\alpha$ from the negative $x$ axis, the envelope equations have the form

$$
\begin{aligned}
& \frac{d^{2} a}{d s^{2}}+\frac{1}{\beta} \frac{d \beta}{d s} \frac{d a}{d s}= \pm \frac{B^{\prime}}{[B \rho]} a+\frac{\epsilon_{x}^{2}}{a^{3}}+\frac{2 K}{a+b}+\frac{f_{0} K}{R^{2}} a-(a \cos \alpha+b \sin \alpha) \frac{\cos \alpha}{\rho^{2}} \\
& \frac{d^{2} b}{d s^{2}}+\frac{1}{\beta} \frac{d \beta}{d s} \frac{d b}{d s}=\mp \frac{B^{\prime}}{[B \rho]} b+\frac{\epsilon_{y}^{2}}{b^{3}}+\frac{2 K}{a+b}-\frac{f_{0} K}{R^{2}} b-(a \cos \alpha+b \sin \alpha) \frac{\sin \alpha}{\rho^{2}} .
\end{aligned}
$$

Here, $a$ and $b$ are the beam coordinate-space radii respectively in the $x$ and $y$ directions. The quadrupolefield transverse gradient is denoted by $B^{\prime}$, with the sign being determined by the magnet orientation. The "magnetic rigidity" in the quadrupole-focusing terms is given in Sl units by $[B \rho]=\beta \gamma M c / q e$, where $M$ and $q$ are the ion mass and charge state, $\beta$ is the axial velocity scaled by the speed of light $c$, and $\gamma M c^{2}$ is the total energy of beam ions. The perveance $K$ in the space-charge and image-force terms is defined as

$$
K=\frac{1}{4 \pi \epsilon_{0}} \frac{2 q e I_{b}}{(\beta \gamma)^{3} M c^{3}}
$$

where $I_{b}$ is the beam current in Amperes, and $\epsilon_{0}$ is the free-space permittivity. The beam transverse temperature is accounted for in Eq. (1) by the terms proportional to the unnormalized emittances $\epsilon_{x}$ and $\epsilon_{y}$, which are calculated here by assuming that the normalized-emittance components $\beta \gamma \epsilon_{x}$ and $\beta \gamma \epsilon_{y}$ are constant. Following the method in Ref. 3, the image-force terms in Eq. (1) were derived by assuming that the centroid of the elliptical beam is displaced a distance $\left(X^{2}+Y^{2}\right)^{1 / 2} \ll R$ from the axis of a straight beam pipe, where $X$ and $Y$ are the beam centroid coordinates. With this assumption, the coefficient $f_{0}$ has the form

$$
f_{0}(a, b, X, Y)=\frac{a^{2}-b^{2}}{4 R^{2}}+\frac{X^{2}-Y^{2}}{R^{2}}+\frac{3}{2} \frac{X^{2}+Y^{2}}{R^{2}}\left(\frac{a^{2}-b^{2}}{R^{2}}\right)+\frac{3}{8} \frac{X^{2}-Y^{2}}{R^{2}}\left(\frac{a^{2}-b^{2}}{R^{2}}\right)^{2} .
$$

The presumption of a straight beam pipe substantially simplifies the algebra and is appropriate when the bend radius $\rho$ is much larger than $R$. The $d \beta / d s$ term in Eq. (1) arises from changing variables from $t$ to $s$, and, as discussed below, an approximate expression for the derivative is obtained directly from one of the equations for longitudinal motion.

Equation for the lattice-plane centroid coordinates $X$ and $Y$ are obtained from distribution averages of the single-particle motion equations. We find

$$
\begin{aligned}
& \frac{d^{2} X}{d s^{2}}+\frac{1}{\beta} \frac{d \beta}{d s} \frac{d X}{d s}= \pm \frac{B^{\prime}}{[B \rho]} X+\left(\frac{\cos \alpha}{\rho}-\frac{B_{d y}}{[B \rho]}\right)+\frac{\left(1+g_{0}\right) K}{R^{2}} X+\frac{g_{1} K}{R^{2}} Y-\left[\frac{X \cos \alpha+Y \sin \alpha}{\rho^{2}}\right] \cos \alpha, \\
& \frac{d^{2} Y}{d s^{2}}+\frac{1}{\beta} \frac{d \beta}{d s} \frac{d Y}{d s}=\mp \frac{B^{\prime}}{[B \rho]} Y+\left(\frac{\sin \alpha}{\rho}+\frac{B_{d x}}{[B \rho]}\right) \\
&: \quad+\frac{\left(1-g_{0}\right) K}{R^{2}} Y+\frac{g_{1} K}{R^{2}} X-\left[\frac{X \cos \alpha+Y \sin \alpha}{\rho^{2}}\right] \sin \alpha,
\end{aligned}
$$


where $\underset{\sim}{B}=B_{d x} \hat{x}+B_{d y} \hat{y}$ is the dipole field, and the image-force coefficients $g_{0}$ and $g_{1}$ are given in the straight-pipe paraxial limit by

$$
\begin{aligned}
& g_{0}(a, b, X, Y)=\frac{a^{2}-b^{2}}{4 R^{2}}+\frac{\left(X^{2}-Y^{2}\right)}{R^{2}}+\frac{3}{4} \frac{\left(X^{2}+Y^{2}\right)}{R^{2}}\left(\frac{a^{2}-b^{2}}{R^{2}}\right) \\
&+\frac{1}{8} \frac{\left(X^{2}-Y^{2}\right)}{R^{2}}\left(\frac{a^{2}-b^{2}}{R^{2}}\right)^{2} \\
& g_{1}(a, b, X, Y)=\frac{2 X Y}{R^{2}}\left[1-\frac{1}{8}\left(\frac{a^{2}-b^{2}}{R^{2}}\right)^{2}\right] .
\end{aligned}
$$

To avoid deflecting the beam from the pipe axis, the dipole-field components should be $B_{d x}=-B_{d} \sin \alpha$ and $B_{d y}=B_{d} \cos \alpha$.

\section{Longitudinal Dynamics}

To model axial dynamics, we treat slices of the beam as Lagrangian fluid elements characterized by an axial velocity $\beta c$ and the time $\tau$ that the slice arrives at an axial location $s$. This approach implicitly assumes that the beam has a negligible longitudinal temperature and that the slices remain approximately collinear. If the slice boundaries are presumed to remain perpendicular to the beam-pipe axis, then the equation for $\tau$ is found from orbit kinematics to be

$$
\frac{d \tau}{d s}=\frac{1}{\beta c}\left(1+\frac{X \cos \alpha+Y \sin \alpha}{\rho}\right),
$$

where we have again assumed paraxial motion. An approximate $\beta$ equation is obtained by retaining only the electrostatic force in the single-particle motion equations and averaging the axial component over the beam elliptical cross-section:

$$
\frac{d \beta}{d s}=\frac{q e}{\beta M c^{2}}\left(1+\frac{X \cos \alpha+Y \sin \alpha}{\rho}\right)\left(E_{e x t}+E_{s c}\right) .
$$

Here, the average external electric field $E_{\text {ext }}$ is approximated only by the voltage across accelerating modules divided by the gap length. The space-charge field is approximated by

$$
E_{s c} \approx g\left[\frac{\partial}{\partial \tau}\left(\frac{\lambda}{\beta c}\right)+\frac{\lambda}{\beta} \frac{d \beta}{d s}\right],
$$

where the line-charge density $\lambda$ for a slice containing charge $\delta Q$ is estimated by

$$
\lambda=\frac{\delta Q}{\beta c \delta \tau},
$$

and the inductance-like factor $g$ is given by

$$
g \approx \frac{1}{4 \pi \epsilon_{0}} \ln \left(\frac{R^{2}}{a b}\right),
$$

provided the beam has a uniform charge in the transverse plane. In deriving the space-charge field, the radial electrostatic field is assumed to vary over a much shorter scale length than $E_{s c}$, and the continuity equation is used to convert derivatives with respect to $s$ into $\tau$ derivatives. When Eq. (8) is substituted into the $\beta$ equation Eq. (7), the resulting equation is trivially rearranged to give an equation for $d \beta / d s$ in terms of $E_{\text {ext }}$ and the time derivative of $\lambda / \beta$.

The equations Eqs. (1) - (9) are recast in the code as a set of ten first-order equations and are integrated by a conventional fourth-order Runge-Kutta method. A constant step size in $s$ is used except near the 
boundaries of lattice elements, where the step is chosen to land on each boundary. The results are found to be insensitive to the choice of step size so long as there are 10 or more integration steps per lattice element. To initialize the equations in equilibrium, we integrate the equations over the first full lattice period and use a vector form of Newton's method to adjust the initial values of the beam radii and their derivatives in each slice until they equal the corresponding final values.

\section{User Interface}

An important aspect of the code is the lattice specification. The user may specify an arbitrary number of distinct lattice elements, specifying such properties as length, aperture, strength, bend angle, and the rotation angle in the plane transverse to the beam direction. At present, the element types allowed in the code are drifts, accelerating gaps, sector bend magnets, electrostatic and magnetic quadrupoles, beamposition monitors, and steering stations. Solenoids and higher-order multipoles might also be added, but the assumptions of the model would have to be substantially modified. Each lattice element is given a name by the user, and lattice sections may be defined by listing names of previously defined elements and subsections along with the number of times the listed items are repeated in that section. The final such grouping of subsections is treated as the complete lattice.

To facilitate code use, the code has a lattice "self-design" option. The main assumption used to modify the lattice parameters is that energy is gained linearly in distance $s$ along the lattice. With this assumption, the code can set both the time-averaged voltage across accelerating gaps and the field strengths of any bend magnets. Compensation for longitudinal space charge is possible by imposing an appropriate time variation on the accelerating voltage. In the code, these voltage "ears" are calculated from appropriate numerical derivatives of the beam current and velocity and can optionally be modified to mimic the effects of the pulse-forming lines or field-effect transistor switches that are usually used to generate the waveforms. In addition, CIRCE has a palette of errors in the strength and alignment of magnets, the timing of acceleration fields, and the position reading of monitors that can be introduce to study error sensitivities. These run options, as well as the beam and lattice specifications, can be entered through either a namelist input file or a graphical user interface.

\section{APPLICATION TO ILSE}

The parameters and acceleration schedule of ILSE require modifications to the CIRCE lattice-design algorithm. The code was originally written to model the recirculating HIF accelerator studied by the Lawrence Livermore National Laboratory. ${ }^{4}$ As proposed, this "recirculator" consists of several circular lattices in which pulses would be focused by superconducting quadruples as their energy increased by about an order of magnitude. The quadrupole occupancy and strength as well as the lattice period would be constant around each ring, and compression would occur during the transfer lines between rings and in a final drift-compression section. In contrast, the lattice parameters in ILSE will change along the accelerator to maintain the desired phase advance and transverse size, and the beam is continually compressed during acceleration. In addition, the beam combiner and achromatic bena have more complicated design requirements than are presently considered in the lattice-design section. Simple algorithms to handle these ILSE lattice-design functions and beam manipulations have been developed and are presented in the following subsections.

\section{lattice transitions}

The current ILSE design has several changes in the lattice period and the strength and occupancy of quadrupoles in order to maintain the transverse focusing during acceleration. To avoid mismatch oscillations, which invariably increase the emittance, we need a criterion for keeping the beam near transverse equilibrium at these lattice transitions. A simple criterion is obtained by noting that, for a beam in equilibrium, the beam charge density $\rho$ depends only on lattice quantities and, for electrostatic focusing, the axial velocity. This result can be derived from the envelope equation $\mathrm{Eq}$. (1) in the continuum limit, in which $a$ and $b$ are replaced by $\langle a\rangle$, their average value over a lattice period. Assuming $\langle a\rangle$ to be constant in $s$ and neglecting the small image and bend terms, we obtain a quadratic equation for $\langle a\rangle$, which in the limit of negligible 

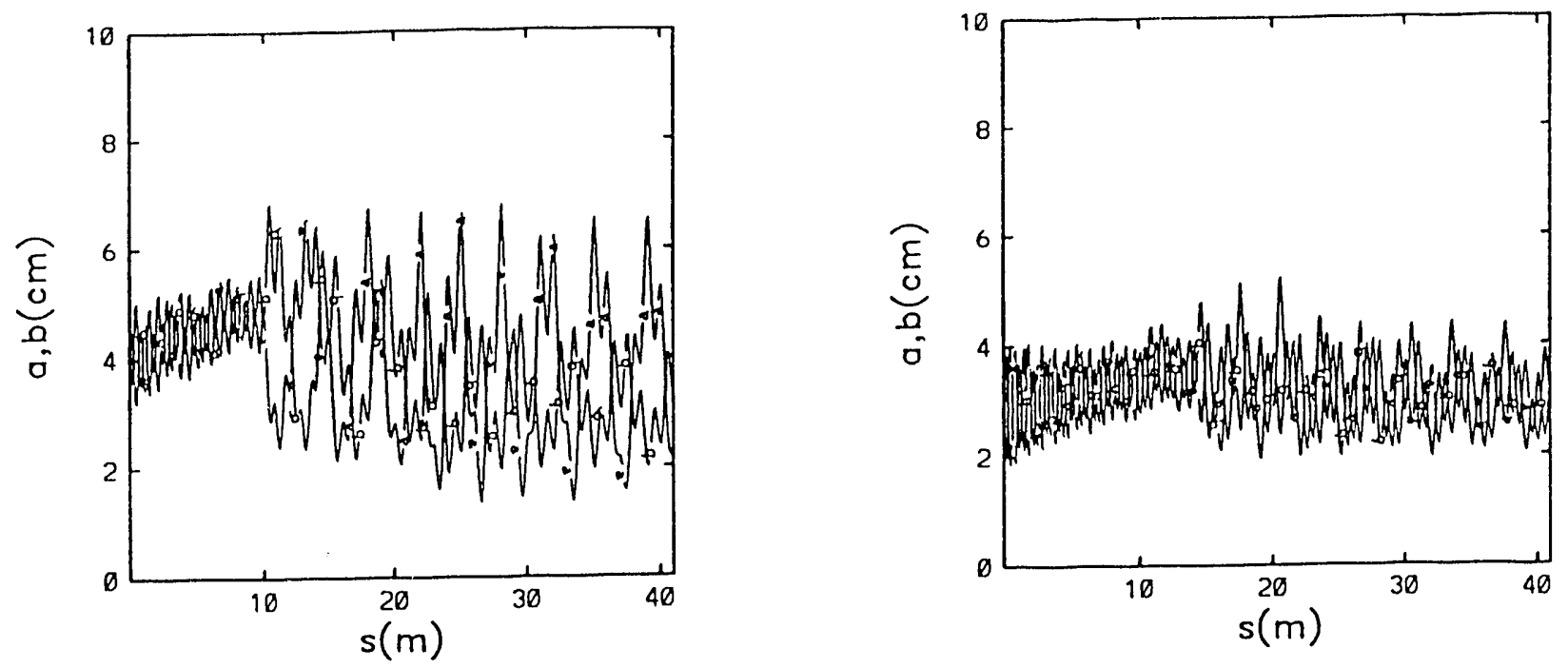

Fig. 1 Radii of the ILSE beam through the acceleration section, calculated by CIRCE, for lattices (a) discontinuous changes in $\eta|\kappa| L$ at three positions and (b) smoothly varying $\eta|\kappa| L$.

emittance gives

$$
\langle a\rangle^{2} \approx \frac{K}{k_{\beta}^{2}},
$$

where $k_{\beta}$ is the betatron wavenumber in the absence of space charge. This assumption of negligible emittance clearly fails near the beam ends, where the perveance vanishes, but it is typically valid over much of the beam. Eq. (11) is put into a usable form by substituting the perveance expression Eq. (2) and using $k_{\beta}^{2} \approx q e \eta_{q} \kappa L /(2 \gamma \beta M c)$, where $\eta_{q}$ is the fraction of the half-lattice period $L$ occupied by the focusing quadrupole. The resulting equation can be rearranged to give

$$
\rho \approx \frac{\epsilon_{0}}{2} \frac{q e \gamma}{M} \eta^{2} \kappa^{2} L^{2}
$$

where we have used the relation $I_{b} \approx \pi\langle a\rangle^{2} \rho \beta c$. This $\rho$ expression suggests that a matched condition, with constant or slowly varying charge density, can be maintained by keeping $\eta_{q}|\kappa| L$ constant across lattice transitions where one or more of the factors change abruptly. This criterion is physically reasonable because $\eta_{q}|\kappa| L$ is proportional to the integrated focusing force in a half lattice period.

The effectiveness of this matching criterion is seen be comparing the CIRCE results in Fig. 1. The beam radii $a$ and $b$ for the center beam slice are shown in Fig. 1a for a preliminary ILSE lattice, constructed from considerations of phase advance and beam radius only, and significant mismatches are seen to occur at the first two points where the lattice period and the quadrupole strength and occupancy are changed. The corresponding plot is shown in Fig. $\mathrm{Ib}$ for a lattice with similar changes in $\eta_{q}$ and $L$, but with the strength chosen to keep $\eta_{q}|\kappa| L$ constant. The latter case, while still not perfectly matched, at least shown no major disruption at the transitions.

\section{beam compression}

A procedure for compressing a beam while maintaining an approximately self-similar axial density profile can be inferred from the longitudinal equations. Neglecting bend terms in Eqs. (6) and (7) and the $d \beta / d s$ term in Eq. (8), we may write the longitudinal equations in the form

$$
\frac{d \tau}{d s} \approx \frac{1}{\beta c}
$$




$$
\frac{d \beta}{d s} \approx \frac{q e}{\beta M c^{2}}\left[E_{e x t}+g \frac{\partial}{\partial \tau}\left(\frac{\lambda}{\beta c}\right)\right] .
$$

The external field $E_{\text {ext }}$ is taken to be the sum of a time-independent acceleration field $E_{a c c}$, a compression field $E_{\text {comp }}$ that typically has a linear variation in time, and a componeni with a more complicated time dependence, termed an "ear field" here, that on average balances the longitudinal space-charge force. The ear field may be written

$$
E_{\text {ear }} \approx-\frac{g}{\eta_{q} \bar{\beta} c} \frac{\partial \lambda}{\partial \tau}
$$

where $\bar{\beta}$ is a density-weighted average of $\beta$. Since this field approximately cancels the space-charge field arising from variation in $\lambda$, the only force between acceleration gaps is due to the remaining $\partial \beta / \partial \tau$ term in Eq. (13b). If we assume that this terms is approximately constant along the beam, than Eq. (13) yields equations between gaps for the velocity variation $c \Delta \beta$ from head to tail and the beam duration $\Delta \tau$ :

$$
\begin{aligned}
& \frac{d \Delta \tau}{d s} \approx-\frac{\Delta \beta}{\bar{\beta}^{2} c} \\
& \frac{d \Delta \beta}{d s} \approx-\frac{g q e Q}{M(\bar{\beta} c)^{4}} \frac{\Delta \beta}{(\Delta \tau)^{2}} .
\end{aligned}
$$

Here, $\lambda$ has been written in terms of the total charge $Q$ using the linear approximation $\lambda \approx Q /(\bar{\beta} c \Delta \tau)$. These equations can be solved exactly, but more useful expressions for $\Delta \beta$ and $\Delta \tau$ are obtained by assuming that the change in $\Delta \tau$ between gaps is small compared with the initial value $\Delta \tau_{0}$ :

$$
\begin{aligned}
& \frac{\Delta \tau}{\Delta \tau_{0}} \approx 1-\frac{\Delta \beta_{0}}{\beta} \frac{s-s_{0}}{\tilde{\beta} c \Delta \tau_{0}} \\
& \frac{\Delta \beta}{\tilde{\beta}} \approx \frac{\Delta \beta_{0}}{\bar{\beta}}\left[1-\frac{g q e \lambda_{0}}{M(\tilde{\beta} c)^{2}} \frac{s-s_{0}}{\bar{\beta} c \Delta \tau_{0}}\right] .
\end{aligned}
$$

The zero subscripts here denote beam quantities at the end of the previous acceleration gap.

With these expressions for the change in $\Delta \beta$ and $\Delta \tau$ between gaps, a simple procedure may be followed to specify suitable compression and ear fields for succeeding gaps. Starting with an initial velocity tilt $\Delta \beta_{0}$ and beam duration $\Delta \tau_{0}$, the beam is advanced to the next gap, and expected values of $\Delta \beta$ and $\Delta \tau$ there are calculated from Eq. (16). Also, the new average velocity after the next gap is estimated from $\bar{\beta} \approx\left(\bar{\beta}_{0}^{2}+q e E_{a c c} \eta_{q} L / M c^{2}\right)^{1 / 2}$. With these expressions, an appropriate ear field at the next gap is given by

$$
E_{e a r} \approx-\frac{g}{g_{0}} \frac{\eta_{q 0}}{\eta_{q}}\left(\frac{\bar{\beta}_{0} \Delta \tau_{0}}{\bar{\beta} \Delta \tau}\right)^{2} E_{e a r 0}\left(\frac{\tau-\bar{\tau}}{\Delta \tau}\right)
$$

where self-similar compression has been assumed. The quantity $\bar{\tau}$ in Eq. (17) is the temporal midpoint of the pulse at the center of the next gap. The linearly varying compression field $E_{c o m p}$ needed to produce a velocity tilt $\Delta \beta_{n e w}$, which is arbitrary so long as $E_{\text {comp }}$ remains physically reasonable, is similarly given by

$$
E_{\text {comp }} \approx \frac{\left(\bar{\beta}+\bar{\beta}_{0}\right) M c}{2 q e \eta_{q} L}\left(\Delta \beta_{\text {new }}-\Delta \beta\right)\left(\frac{\tau-\bar{\tau}}{\Delta \tau}\right)
$$

This calculation is repeated at each succeeding gaps.

The principle shortcoming of this procedure is the absence of any overall compression schedule. The allowable beam duration at any point in the accelerator is obviously tied to the focusing strength and the beam-pipe radius, but the correlation has not been worked out. Also, a modified procedure for shaping the pulse prior to final compression must be developed.

beam combination 

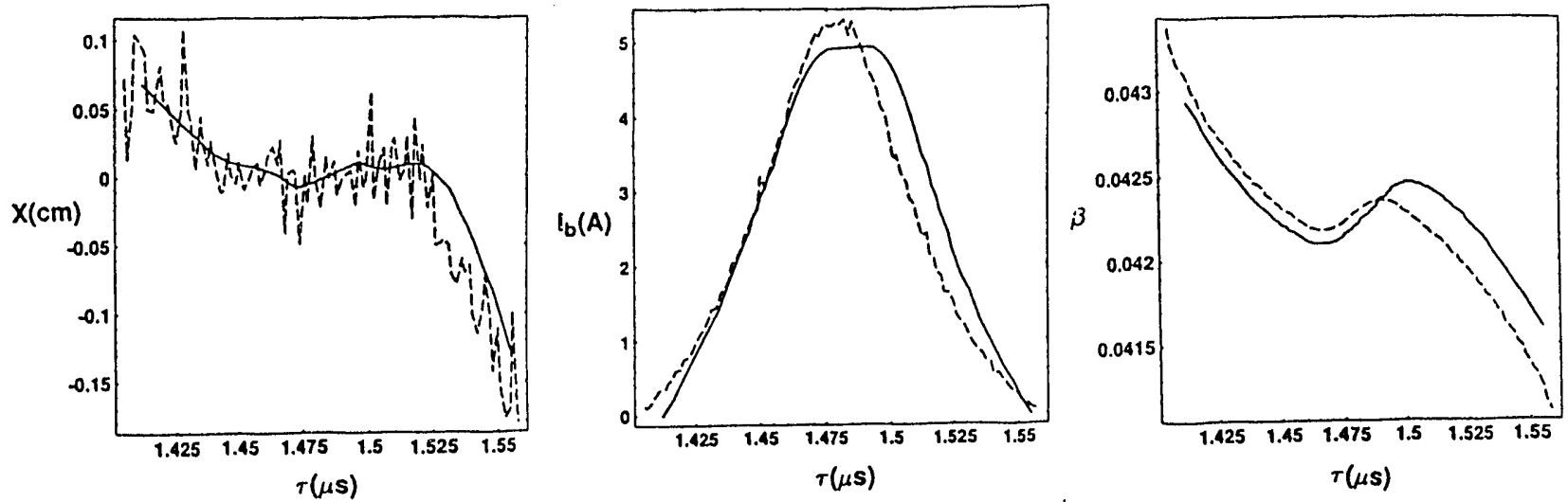

Fig. 2 ILSE beam quantities after traversing a $180^{\circ}$ achromatic bend, as calculated by CIRCE (solid line) and WARP3d (dashed line): (a) current; (b) axial velocity scaled by $c$; (c) displacement in $x$.

As presently conceived, the beam combiner will consist of a series of bends and quadrupole lenses that finally arranges the four ILSF, beams into an approximation of a circular cross section. The resulting single beam would then have four times the current of each of the original beams, slightly more than four times the original emittance, and roughly twice the radius. The detailed beam dynamics in this section is difficult to model using CIRCE due to the complicated geometry, the inter-beam forces prior to merging, and the enhanced emittance growth resulting from entrapment of low-density regions of phase space. Instead, the combiner is initially being modeled in the code as a zero-length lattice element in which the current and emittance are simply multiplied by the number of beams $N_{b}$ being combined, and the radii $a$ and $b$ are multiplied by $N_{b}^{1 / 2}$.

achromatic bend

The ILSE achromatic bend is intended to change the beam direction by $180^{\circ}$ while approximately preserving the phase-space distribution of ions over some range of $\beta$ values. This achromaticity is necessary because the beam is planned to have a residual head-to-tail velocity variation after acceleration of a few percent. Although achromats can readily be designed in the absence of image forces, no systematic procedure including these forces has been devised for beams whose transv rrse dynamics is dominated by space charge.

To design an achromatic bend for a space-charge dominated beam such as that in ILSE, we plan to adapt an optimization routine developed by Hewett and Bangerter. ${ }^{5}$ The technique, which is akin to a multidimensional Muller's method, in principal allows an arbitrary number of lattice variables to be adjusted to optimally match user-specified performance criteria. For the ILSE bend, quadrupole strengths and positions could be adjusted to minimize final centroid displacements and transverse velocities of beam slices with initial axial velocities above, at, and below the design velocity. A supplemental criterion would also require that the greatest transverse excursion of the beam slices be simultaneously minimized.

At present, the bend lattice specified in CIRCE is achromatic through first order in position and momentum in the absence of image forces. With this choice, we find that a $10 \mathrm{MeV}$ beam of ${ }^{12} \mathrm{C}$ ions with a linear $3 \%$ velocity increase from head to tail develops the transverse displacement shown by the solid curve in Fig. $2 \mathrm{a}$. For this case, the peak beam current increases by $46 \%$ due the the velocity tilt to a final value of $5.1 \mathrm{~A}$, as shown in Fig. $2 \mathrm{~b}$, while the longitudinal space charge increases the beam duration by $17.5 \%$. 
The plot of $\beta$ for the beam slices in Fig. 2c shows that the beam center is still compressing at the end of the bend, but the ends are expanding due to the longitudinal space charge. The corresponding plots from the three-dimensional particle simulation WARP3d are shown as dashed lines in Figs. 2a-c, with the transverse average of $\beta$ being plotted in Fig. 2c. The differences between the results are seen to be minor and to occur near the beam ends, where the codes use somewhat different algorithms to initialize the beam. The good agreement here between CIRCE and WARP3d is nontrivial because the assumptions in CIRCE of zero longitudinal temperature and perpendicular slice boundaries are visibly violated in the WARP3d simulation.

\section{REFERENCES}

1 T. Fessenden, R. Bangerter, D. Berners, J. Chew, S. Eylon, A. Faltens, W. Fawley, C. Fong, M. Fong. K. Hahn, E. Henestroza, D. Judd, E. Lee, C. Lionberger, S. Mukherjee, C. Peters, C. Pike, G. Raymond, L. Reginato, H. Rutkowski, P. Siedl, L. Smith, D. Vanecek, S. Yu, F. Deadrick, A. Friedman, L. Griffith, D. Hewett, M. Newton, and H. Shay, "ILSE, The Next Step toward a Heavy Ion Induction Accelerator for Inertial Fusion Energy," Proc. 14th Int. Conf. on Plasma Physics and Controlled Nuclear Fusion Research, IAEA, Wurzburg, Germany, 30 September - 7 October 1992.

2 W. M. Sharp, J. J. Barnard, and S. S. Yu, Part. Accel. 37-38, 205 (1992).

3 E. P. Lee, E. Close, and L. Smith, "Space Charge Effects in a Bending Magnet System," in Proceeding of the 1987 IEEE Part. Accel. Conf, E. R. Lindstrom and L. S. Taylor, eds. (IEEE, New Jersey, 1987), p. 1126.

4 J. J. Barnard, A. L. Brooks, J. P. Clay, F. E. Coffield, F. J. Deadrick, L. V. Griffith, A. R. Harvey, D. L. Judd, H. C. Kirbie, V. K. Neil, M. A. Newton, A. C. Paul, L. L. Reginato, G. E. Russell, W. M. Sharp, H. D. Shay, J. H. Wilson, and S. S. Yu, "Study of Recirculating Induction Accelerators as Drivers for Heavy Ion Fusion," Lawrence Livermore National Laboratory Report UCRL-LR-108095, May 1992.

5 D. W. Hewett and R. O. Bangerter, private communication. 

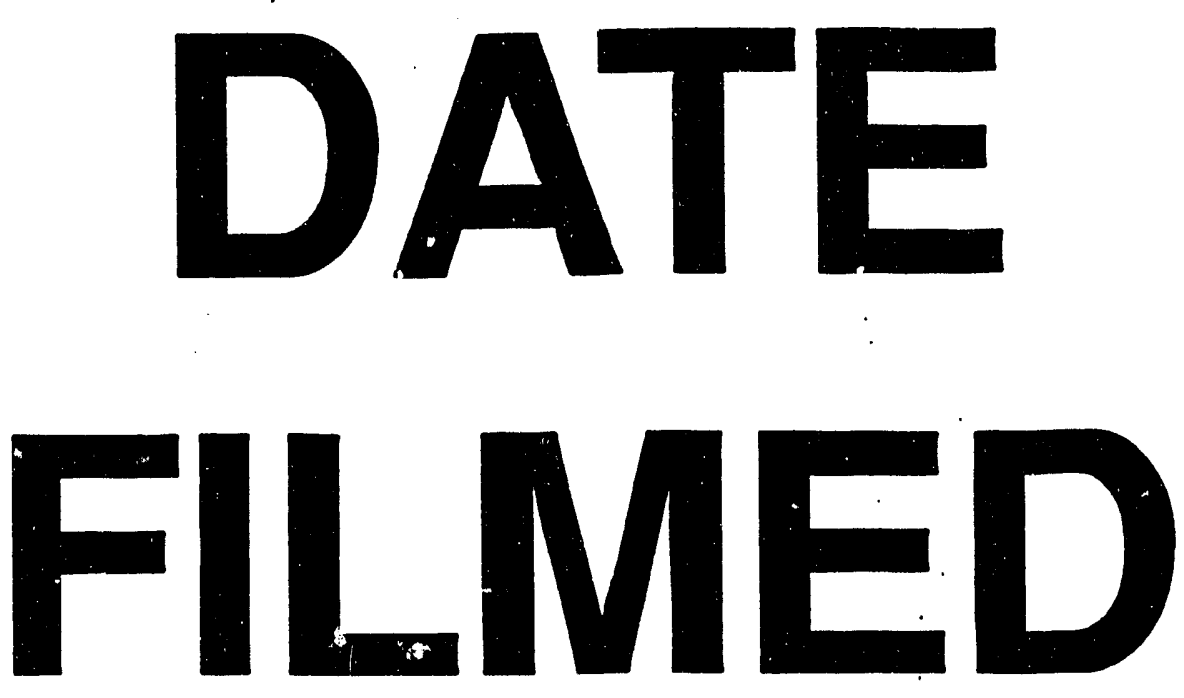

$9 / 16 / 93$
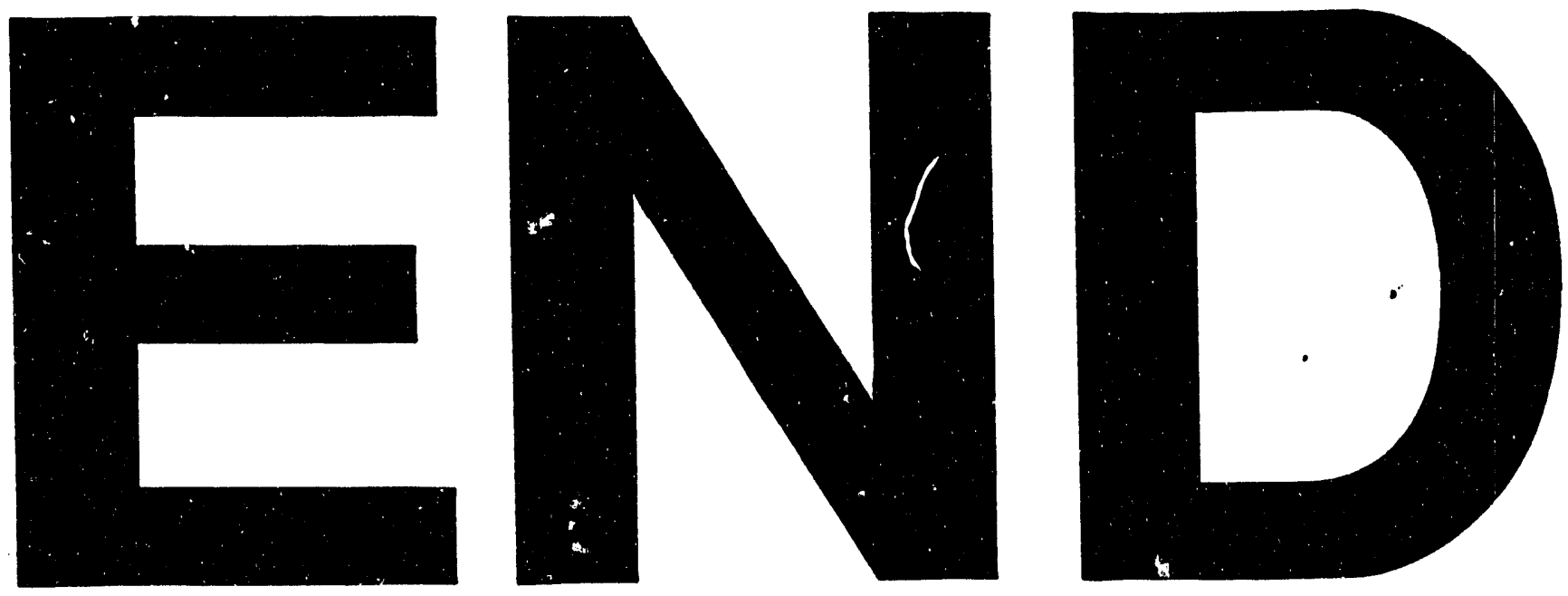
